\title{
Structure of long idempotent-sum free sequences over finite cyclic semigroups
}

\author{
Guoqing Wang \\ Department of Mathematics, Tianjin Polytechnic University, Tianjin, 300387, P. R. China \\ Email: gqwang1979@aliyun.com
}

\begin{abstract}
Let $\mathcal{S}$ be a finite cyclic semigroup written additively. An element $e$ of $\mathcal{S}$ is said to be idempotent if $e+e=e$. A sequence $T$ over $\mathcal{S}$ is called idempotent-sum free provided that no idempotent of $\mathcal{S}$ can be represented as a sum of one or more terms from $T$. We prove that an idempotent-sum free sequence over $\mathcal{S}$ of length over approximately a half of the size of $\mathcal{S}$ is well-structured. This result generalizes the Savchev-Chen Structure Theorem for zero-sum free sequences over finite cyclic groups.
\end{abstract}

Key Words: Idempotent-sum free sequences; Zero-sum free sequences; Zero-sum; Inverse zero-sum problems; Idempotents; Cyclic semigroups

\section{Introduction}

Let $G$ be a finite cyclic group of order $n$, and let $T$ be a sequence of terms from $G$. We say that $T$ is zero-sum free, if no nonempty subsequence of $T$ sums to zero (the identity element of $G$ ). An easy observation shows that $n-1$ is the maximal length of a zero-sum free sequence and if $T$ is a zero-sum free sequence of length exactly $n-1$, then $T$ consists of one element $g$ which is repeated $n-1$ times. Investigations of the structure of long zero-sum free sequences were started in 1970s' by J.D. Bovey, P. Erdôs and I. Niven [1], who proved that any long zero-sum free sequence of length $\ell$ over the cyclic group of order $n$ must contain one term with repetitions at least $2 \ell-n+1$. After that, the study of the structure of long zero-sum free sequences over cyclic groups has attracted considerable attention (see [7, 8, 13, 16, 21, 27]), among which for a cyclic group of order $n, \mathrm{~W}$. Gao [7] characterized the zero-sum free sequence of length roughly greater than $\frac{2 n}{3}$, S. Savchev and F. Chen [21] proved that each zero-sum free sequence with 
length greater than $\frac{n}{2}$ must have a regular structure. The Savchev-Chen structural zero-sum theorem (see [19], Chapter 11) found a variety of applications in problems of zero-sum theory.

Problems of this flavor are known as inverse zero-sum problems, which ask for an structural description of sequences without the desired prescribed properties. Often the study of inverse problems is complex. For example, even for the simplest noncyclic case-rank 2 groups (the direct sum of two finite cyclic groups), it is only recently, and with a massive amount combined efforts of W. Gao, A. Geroldinger, D. Grynkiewicz and W.A. Schmid in several multi-pages articles (see [9-12, 22]), and of C. Reiher [20] in a final step, that the structure of zero-sum free sequences of the maximal length has been determined. Besides of being of interest for its own right, the inverse zero-sum problems are particularly relevant to the theory of non-unique factorizations. For this connection and some general information on factorization theory we refer to $[5,14,15]$.

Another motivation of this manuscript comes from the question (see [4, 15]) proposed by P. Erdôs to D.A. Burgess which can restated as follows:

"Let $\mathcal{S}$ be a finite semigroup of order $n$. Is there an idempotent-product free sequence of terms from $\mathcal{S}$ with length $n$ ?"

A sequence $T$ over $\mathcal{S}$ is called idempotent-product free provided that no idempotent of $\mathcal{S}$ can be represented as a product of one or more terms from $T$ in any order. The Erdôs' question was answered partially by Burgess [2] in the case when $\mathcal{S}$ is commutative or contains only one idempotent, and was completely affirmed by D.W.H. Gillam, T.E. Hall and N.H. Williams [17], and was extended to infinite semigroups by the author [25]. Note that if the semigroup $\mathcal{S}$ is commutative, we use idempotent-sum free for idempotent-product free since the operation is addition and the order of terms in additions does not matter. In particular, if $\mathcal{S}$ is a finite abelian group, the notion idempotent-sum free reduces to be zero-sum free, because the identity element is the unique idempotent in a group.

Recently some zero-sum type problems were investigated in the setting of commutative semigroups (see $[6,23,24,26,28]$ for example). In this manuscript we show that an idempotentsum free sequence over a finite cyclic semigroup of length over approximately a half of the size of the cyclic semigroup will yield a regular structure, which generalizes the Savchev-Chen Structure Theorem for zero-sum free sequences over finite cyclic groups. Moreover, we investigate the Ramsey-type question to determine the least integer $\ell$ to ensure that an idempotent-sum free sequence of length at least $\ell$ will have this regular structure, meanwhile, we also extend an invariant proposed by S.T. Chapman, M. Freeze and W.W. Smith [3, 4] on minimal zero-sum sequences into finite cyclic semigroups. 


\section{Notation and terminologies}

For integers $a, b \in \mathbb{Z}$, we set $[a, b]=\{x \in \mathbb{Z}: a \leq x \leq b\}$. For a real number $x$, we denote by $\lfloor x\rfloor$ the largest integer that is less than or equal to $x$, and by $\lceil x\rceil$ the smallest integer that is greater than or equal to $x$.

Let $\mathcal{S}$ be a commutative semigroup written additively, where the operation is denoted as + . For any positive integer $m$ and any element $a \in \mathcal{S}$, we denote by $m a$ the sum $\underbrace{a+\cdots+a}_{m}$. An element $e$ of $\mathcal{S}$ is said to be idempotent if $e+e=e$. A cyclic semigroup is a semigroup generated by a single element $s$, denoted $\langle s\rangle$, consisting all elements which can be represented as $m s$ for some positive integer $m$. If the cyclic semigroup $\langle s\rangle$ is infinite then $\langle s\rangle$ is isomorphic to the semigroup of $\mathbb{N}$ with addition (see [18], Proposition 5.8), and if $\langle s\rangle$ is finite then the least integer $k>0$ such that $k s=t s$ for some positive integer $t \neq k$ is called the index of $\langle s\rangle$, then the least integer $n>0$ such that $(k+n) s=k s$ is called the period of $\langle s\rangle$. We denote a finite cyclic semigroup of index $k$ and period $n$ by $C_{k ; n}$. In particular, if $k=1$ the semigroup $C_{k ; n}$ reduces to a cyclic group of order $n$. For any element $a$ of $C_{k ; n}=\langle s\rangle$, let $\operatorname{ind}_{s}(a)(\operatorname{write}$ as ind $(a)$ for simplicity) be the least positive integer $t$ such that $t s=a$. Let $\mathbb{Z} / n \mathbb{Z}$ be the additive group of integers modulo $n$. Define a map

$$
\psi: C_{k ; n} \rightarrow \mathbb{Z} / n \mathbb{Z} \quad \text { given by } \quad \psi: a \mapsto \operatorname{ind}(a)+n \mathbb{Z} \quad \text { for all } a \in C_{k ; n} .
$$

We also need to introduce notation and terminologies on sequences over semigroups and follow the notation of A. Geroldinger, D.J. Grynkiewicz and others used for sequences over groups (cf. [[19], Chapter 10] or [[15], Chapter 5]). Let $\mathcal{F}(\mathcal{S})$ be the free commutative monoid, multiplicatively written, with basis $\mathcal{S}$. We denote multiplication in $\mathcal{F}(\mathcal{S})$ by the boldsymbol and we use brackets for all exponentiation in $\mathcal{F}(\mathcal{S})$. By $T \in \mathcal{F}(\mathcal{S})$, we mean $T$ is a sequence of terms from $\mathcal{S}$ which is unordered, repetition of terms allowed. Say $T=a_{1} a_{2} \cdot \ldots \cdot a_{\ell}$ where $a_{i} \in \mathcal{S}$ for $i \in[1, \ell]$. The sequence $T$ can be also denoted as $T=\underset{a \in \mathcal{S}}{\bullet} a^{\left[\mathrm{v}_{a}(T)\right]}$, where $\mathrm{v}_{a}(T)$ is a nonnegative integer and means that the element $a$ occurs $\mathrm{v}_{a}(T)$ times in the sequence $T$. By $|T|$ we denote the length of the sequence, i.e., $|T|=\sum_{a \in \mathcal{S}} \mathrm{v}_{a}(T)=\ell$. By $\varepsilon$ we denote the empty sequence over $\mathcal{S}$ with $|\varepsilon|=0$. We call $T^{\prime}$ a subsequence of $T$ if $\mathrm{v}_{a}\left(T^{\prime}\right) \leq \mathrm{v}_{a}(T)$ for each element $a \in \mathcal{S}$, denoted by $T^{\prime} \mid T$, moreover, we write $T^{\prime \prime}=T \cdot T^{[-1]}$ to mean the unique subsequence of $T$ with $T^{\prime} \cdot T^{\prime \prime}=T$. We call $T^{\prime}$ a proper subsequence of $T$ provided that $T^{\prime} \mid T$ and $T^{\prime} \neq T$. In particular, the empty sequence $\varepsilon$ is a proper subsequence of every nonempty sequence. We say $T_{1}, \ldots, T_{m}$ are disjoint subsequences of $T$ provided that $T_{1} \cdot \ldots \cdot T_{m} \mid T$. Let $\sigma(T)=a_{1}+\cdots+a_{\ell}$ be the sum of all terms from $T$. Let $\Sigma(T)$ be the set consisting of the elements of $\mathcal{S}$ that can be represented as a sum of one or more terms from $T$, i.e., $\Sigma(T)=\left\{\sigma\left(T^{\prime}\right): T^{\prime}\right.$ is taken over all nonempty subsequences of $\left.T\right\}$. We call $T$ a zero-sum sequence provided that $\mathcal{S}$ has an identity $0_{\mathcal{S}}$ and $\sigma(T)=0_{\mathcal{S}}$. In particular, if $\mathcal{S}$ has an identity $0_{\mathcal{S}}$, we adopt the convention that $\sigma(\varepsilon)=0_{\mathcal{S}}$. We say the sequence $T$ is 
- a zero-sum free sequence if $T$ contains no nonempty zero-sum subsequence;

- a minimal zero-sum sequence if $T$ is a nonempty zero-sum sequence and and $T$ contains no nonempty proper zero-sum subsequence;

- an idempotent-sum sequence if $\sigma(T)$ is an idempotent;

- an idempotent-sum free sequence if $T$ contains no nonempty idempotent-sum subsequence;

- a minimal idempotent-sum sequence if $T$ is a nonempty idempotent-sum sequence and $T$ contains no nonempty proper idempotent-sum subsequence.

It is worth remarking that when the commutative semigroup $\mathcal{S}$ is an abelian group, the notion zero-sum free sequence and idempotent-sum free sequence make no difference, and the similar holds for the pair of terminologies minimal zero-sum sequence and minimal idempotent-sum sequence.

For a finite cyclic semigroup $C_{k ; n}$, we extend $\psi$ to the map

$\Psi: \mathcal{F}\left(C_{k ; n}\right) \rightarrow \mathcal{F}(\mathbb{Z} / n \mathbb{Z}) \quad$ given by $\quad \Psi: T \mapsto \underset{a \mid T}{\bullet} \psi(a) \quad$ for any sequence $T \in \mathcal{F}\left(C_{k ; n}\right)$.

\section{Structure of long idempotent-sum free sequences}

To give the main theorem, we shall need some preliminaries.

Lemma 3.1. (Folklore) Let $G$ be a cyclic group of order $n \geq 2$. If $T \in \mathcal{F}(G)$ is either a zerosum free sequence of length $n-1$ or a minimal zero-sum sequence of length $n$, then $T=g^{[n-1]}$ or resp. $T=g^{[n]}$ for some $g \in G$ with $\operatorname{ord}(g)=n$.

Lemma 3.2. Let $G$ be a cyclic group of order $n \geq 2$, and let $T \in \mathcal{F}\left(G \backslash\left\{0_{G}\right\}\right)$ be a sequence of length at least $n-1$. Let $U$ be one of the longest zero-sum subsequences of $T$. If $\left|T \cdot U^{[-1]}\right|=n-1$ then $T=g^{[T]]}$ for some $g \in G$ with $\operatorname{ord}(g)=n$.

Proof. We take disjoint minimal zero-sum subsequences of $T$, say $U_{1}, \ldots, U_{\ell}$, with $\ell$ being maximal. Let $U_{0}=T \cdot\left(U_{1} \cdot \ldots \cdot U_{\ell}\right)^{[-1]}$. By the maximality of $\ell$, we have $U_{0}$ is zero-sum free. It follows that $\left|U_{0}\right| \leq \mathrm{D}(G)-1=n-1$, which implies that $U=U_{1} \cdot \ldots \cdot U_{\ell}$ is one of the longest zero-sum subsequence of $T$ and $\left|U_{0}\right|=n-1$. It follows from Lemma 3.1 that

$$
U_{0}=z^{[n-1]}
$$

for some $z \in G$ with $\operatorname{ord}(z)=n$. Now it suffices to assume that $|T| \geq n$, i.e., $\ell>0$, and prove that $\alpha=z$ for any term $\alpha \mid U$. 
Take arbitrary $\theta \in[1, \ell]$ and take an arbitrary term $x$ of $U_{\theta}$. Since $\left|x \cdot U_{0}\right|=n=\mathrm{D}(G)$ and $U_{0}$ is zero-sum free, it follows that $x \cdot U_{0}$ contains a minimal zero-sum subsequence $U_{\theta}^{\prime}$ with $x \mid U_{\theta}^{\prime}$. Let $U_{0}^{\prime}=\left(U_{\theta} \cdot U_{0}\right) \cdot U_{\theta}^{\prime[-1]}$. Observe that $U_{1}, \ldots, U_{\theta-1}, U_{\theta}^{\prime}, U_{\theta+1}, \ldots, U_{\ell}$ are disjoint minimal zero-sum subsequences of $T$, and that $U_{0}^{\prime}=T \cdot\left(U_{1} \cdot \ldots \cdot U_{\theta-1} \cdot U_{\theta}^{\prime} \cdot U_{\theta+1} \cdot \ldots \cdot U_{\ell}\right)^{[-1]}$. By the maximality of $\ell$, we see that $U_{0}^{\prime}$ is also a zero-sum free sequence and

$$
\left|U_{0}^{\prime}\right|=n-1 .
$$

Suppose $\left|U_{\theta}^{\prime}\right|=n$. Then $U_{\theta}^{\prime}=x \cdot U_{0}$. It follows from Lemma 3.1 that $x=z$.

Suppose $\left|U_{\theta}^{\prime}\right|<n$. Observe that $U_{0}^{\prime}=\left(\left(x \cdot U_{0}\right) \cdot U_{\theta}^{[-1]}\right) \cdot\left(U_{\theta} \cdot x^{[-1]}\right)$ and $\left(x \cdot U_{0}\right) \cdot U_{\theta}^{\prime[-1]}$ is a nonempty subsequence of $U_{0}$. Since all terms of $T$ are nonzero, it follows that

$$
\left|U_{\theta}\right|>1
$$

and $\left|U_{\theta} \cdot x^{[-1]}\right|>0$. By (1) and Lemma 3.1, we derive that $y=z$ for every term $y \mid U_{\theta} \cdot x^{[-1]}$. By (2), we have that as $x$ takes every term of $U_{\theta}$, so does $y$. By the arbitrariness of choosing $\theta$ from $[1, \ell]$ and the arbitrariness of choosing $x$ from $U_{\theta}$, we have the lemma proved.

Lemma 3.3. ([18], Chapter I) Let $\mathcal{S}=C_{k ; n}$ be a finite cyclic semigroup generated by the element $s$. Then $\mathcal{S}=\{s, \ldots, k s,(k+1) s, \ldots,(k+n-1) s\}$ with

$$
i s+j s= \begin{cases}(i+j) s, & \text { if } i+j \leq k+n-1 ; \\ t s, & \text { if } i+j \geq k+n, \text { where } k \leq t \leq k+n-1 \text { and } t \equiv i+j \quad(\bmod n) .\end{cases}
$$

Moreover, there exists a unique idempotent, say $\ell s$, in the cyclic semigroup $\langle s\rangle$, where

$$
\ell \in[k, k+n-1] \text { and } \ell \equiv 0 \quad(\bmod n) .
$$

By Lemma 3.3, it is easy to derive the following.

Lemma 3.4. Let $\mathcal{S}=C_{k ; n}$, and let $W \in \mathcal{F}(\mathcal{S})$ be a nonempty sequence. Then $W$ is an idempotent-sum sequence if, and only if, $\sum_{a \mid W} \operatorname{ind}(a) \geq\left\lceil\frac{k}{n}\right\rceil n$ and $\sum_{a \mid W} \operatorname{ind}(a) \equiv 0(\bmod n)$.

Definition 3.5. ([14]], Definition 5.1.3) Let $G$ be an abelian group. Let $T \in \mathcal{F}(G)$ with $T=$ $\left(n_{1} g\right) \cdot \ldots \cdot\left(n_{\ell} g\right)$, where $\ell=|T| \in \mathbb{N}, g \in G, 1=n_{1} \leq \cdots \leq n_{\ell}, n=n_{1}+\cdots+n_{\ell} \leq \operatorname{ord}(g)$ and $\sum(T)=\{g, 2 g, \ldots, n g\}$. If $n<\operatorname{ord}(g)$ we call $T$ smooth (zero-sum free smooth in full), and if $n=\operatorname{ord}(g)$ we call $T$ zero-sum smooth. In the case we say more precisely that $T$ is (zero-sum free) $g$-smooth and zero-sum g-smooth respectively.

We remark that in Definition 5.1.3 of [14], the notion 'smooth' is used only for zero-sum free sequences. To describe both idempotent-sum free sequences and minimal idempotent-sum sequences in this paper, we extend the notion as above and define zero-sum smooth sequences. Note that for any nonempty sequence $T \in \mathcal{F}(\mathbb{Z}), T$ is 1 -smooth if and only if $\sum(T)=\left[1, \sum_{a \mid T} a\right]$. 
Lemma 3.6. For $\ell \geq 1$, let $T=\underset{i \in[1, \ell]}{\bullet} h_{i} \in \mathcal{F}(\mathbb{Z})$ where $h_{i}>0$ for each $i \in[1, \ell]$. Suppose that the sequence $T$ is not 1-smooth. Then $\sum_{i=1}^{\ell} h_{i} \geq 2 \ell$. Moreover, the equality $\sum_{i=1}^{\ell} h_{i}=2 \ell$ holds if and only if either $T=1^{[\ell-1]} \cdot(\ell+1)$ or $T=2^{[\ell]}$.

Proof. By induction on $\ell$. If $\ell=1$, the conclusion is obvious. Hence, we assume that the conclusion holds true for all $\ell<m$ with $m \geq 2$. Consider the case of $\ell=m$.

Suppose $h_{i} \geq 2$ for all $i \in[1, m]$. Then $\sum_{i=1}^{m} h_{i} \geq 2 m$ and equality holds if and only if $T=2^{[m]}$. Hence, we may assume without loss of generality that $h_{1}=1$.

Let $\lambda$ be the largest length of 1 -smooth subsequences of $T$. Notice that

$$
1 \leq \lambda \leq m-1 \text {. }
$$

Say $\underset{i \in[1, \lambda]}{\bullet} h_{i}$ is 1-smooth. Since $\sum\left(\underset{i \in[1, \lambda]}{\bullet} h_{i}\right)=\left[1, \sum_{i \in[1, \lambda]} h_{i}\right]$, it follows from the maximality of $\lambda$ that $h_{t} \geq 2+\sum_{i \in[1, \lambda]} h_{i} \geq 2+\lambda$ for all $t \in[\lambda+1, m]$. Combined with (3), we have $\sum_{i=1}^{m} h_{i}=$ $\sum_{i=1}^{\lambda} h_{i}+\sum_{t=\lambda+1}^{m} h_{t} \geq \lambda+(m-\lambda) *(2+\lambda) \geq 2 m$, moveover, equality $\sum_{i=1}^{m} h_{i}=2 m$ holds if and only if $\lambda=m-1$ and $\sum_{i=1}^{\lambda} h_{i}=\lambda=m-1$ and $h_{m}=2+\lambda=m+1$, equivalently, $T=1^{[m-1]} \cdot(m+1)$.

Lemma 3.7. Let $\mathcal{S}=\mathrm{C}_{k ; n}$ with $k>n \geq 1$. Let $T \in \mathcal{F}(\mathcal{S})$ be a sequence of length at least $\frac{\left(\left\lceil\frac{k}{n}\right\rceil+1\right) n}{2}-1$. Then $T$ is idempotent-sum free if, and only if, one of the following conditions holds:

(i) $\underset{a \mid T}{\bullet} \operatorname{ind}(a)$ is a 1-smooth sequence with $\sum_{a \mid T} \operatorname{ind}(a) \leq\left\lceil\frac{k}{n}\right\rceil n-1$;

(ii) $\underset{a \mid T}{\bullet} \operatorname{ind}(a)=2^{\left[\frac{\left.\left(\frac{k}{n}\right\rceil+1\right) n}{2}-1\right]}$ with $n \geq 3$ and $\left\lceil\frac{k}{n}\right\rceil n \equiv 1(\bmod 2)$;

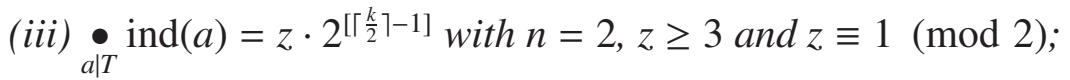

(iv) $\underset{a \mid T}{\bullet} \operatorname{ind}(a)=1^{\left[\frac{k-3}{2}\right]} \cdot \frac{k+1}{2}$ with $n=1$ and $k \equiv 1(\bmod 2)$;

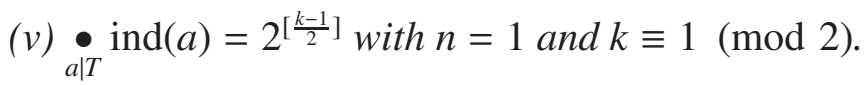

Proof. By Lemma 3.4, it is easy to verify that if the sequence $T$ is given as any one of (i)-(v), then $T$ is idempotent-sum free. Hence, we need only to prove the necessity.

Suppose $\underset{a \mid T}{\bullet} \operatorname{ind}(a)$ is 1 -smooth. Then $\sum(\underset{a \mid T}{\bullet} \operatorname{ind}(a))=\left[1, \sum_{a \mid T} \operatorname{ind}(a)\right]$. Since $T$ is idempotentsum free, it follows that from Lemma 3.4 that $\left\lceil\frac{k}{n}\right\rceil n \notin \sum(\underset{a \mid T}{\bullet} \operatorname{ind}(a))$, and so $\sum_{a \mid T} \operatorname{ind}(a) \leq\left\lceil\frac{k}{n}\right\rceil n-1$. Then (i) holds. Hence, we may assume that $\underset{a \mid T}{\bullet} \operatorname{ind}(a)$ is not 1 -smooth. 
Suppose $n=1$. By Lemma 3.4 and Lemma 3.6, we have that $k-1 \geq \sum_{a \mid T} \operatorname{ind}(a) \geq$ $2|\underset{a \mid T}{\bullet} \operatorname{ind}(a)|=2|T| \geq k-1$, which implies that $\sum_{a \mid T} \operatorname{ind}(a)=2|\underset{a \mid T}{\bullet} \operatorname{ind}(a)|=k-1$ and that either (iv) or (v) holds. Hence, we may assume that

$$
n \geq 2 \text {. }
$$

It follows that

$$
\left.\sum_{a \mid T} \operatorname{ind}(a) \geq 2 \mid \underset{a \mid T}{\bullet \operatorname{ind}(a) \mid \geq 2\left(\left\lceil\frac{k}{n}\right\rceil+1\right) n}-1\right) \geq\left\lceil\frac{k}{n}\right\rceil n .
$$

Claim A. For any nonempty subsequence $L$ of $T$ such that $\sum_{a \mid L} \operatorname{ind}(a) \geq n-1$, the sequence $\bullet \operatorname{ind}(a)$ is not 1-smooth.

Proof of Claim A. Suppose to the contrary that there exists a nonempty subsequence $L$ of $T$ such that $\sum_{a \mid L} \operatorname{ind}(a) \geq n-1$ and $\bullet \operatorname{ind}(a)$ is 1 -smooth. By (5), there exists a subsequence $V$ (perhaps is an empty sequence) of $L$ such that $\sum_{a \mid T \cdot V^{[-1]}} \operatorname{ind}(a) \equiv 0(\bmod n)$ and $\sum_{a \mid T \cdot V^{[-1]}} \operatorname{ind}(a) \geq$ $\left\lceil\frac{k}{n}\right\rceil n$, and thus by Lemma 3.4 $T \cdot V^{[-1]}$ is a nonempty idempotent-sum subsequence of $T$, which contradicts with $T$ being idempotent-sum free. This proves Claim A.

By (4), we have that $|\Psi(T)|=|T| \geq \frac{\left(\left\lceil\frac{k}{n}\right\rceil+1\right) n}{2}-1 \geq \frac{3 n}{2}-1 \geq n=\mathrm{D}(\mathbb{Z} / n \mathbb{Z})$. Let $U$ be a nonempty subsequence of $T$ such that $\Psi(U)$ is a zero-sum sequence over $\mathbb{Z} / n \mathbb{Z}$, i.e., $\sum_{a \mid U} \operatorname{ind}(a)$ is a positive multiple of $n$, with $|U|$ being maximal. Let $W=T \cdot U^{[-1]}$. Then $\Psi(W)$ is either empty or zero-sum free and so $|W|=|\Psi(W)| \leq \mathrm{D}(\mathbb{Z} / n \mathbb{Z})-1=n-1$. Combined with Claim A, Lemma 3.4 and Lemma 3.6, we conclude that

$$
\begin{aligned}
\left(\left\lceil\frac{k}{n}\right\rceil-1\right) n & \geq \sum_{a \mid U} \operatorname{ind}(a) \\
& \geq 2|U|=2(|T|-|W|) \\
& \geq 2(|T|-(n-1)) \\
& \geq 2\left(\frac{\left(\left\lceil\frac{k}{n}\right\rceil+1\right) n}{2}-1-(n-1)\right) \\
& =\left(\left\lceil\frac{k}{n}\right\rceil-1\right) n .
\end{aligned}
$$

It follows that

$$
\begin{gathered}
|W|=n-1, \\
|T|=\frac{\left(\left\lceil\frac{k}{n}\right\rceil+1\right) n}{2}-1,
\end{gathered}
$$

and

$$
\left(\left\lceil\frac{k}{n}\right\rceil-1\right) n=\sum_{a \mid U} \operatorname{ind}(a)=2|U| .
$$


Since $\sum_{a \mid U} \operatorname{ind}(a)$ is a positive multiple of $n$, it follows from Claim A that $\underset{a \mid U}{\bullet} \operatorname{ind}(a)$ is not 1smooth. By Lemma 3.6 and (8), we have that

$$
\underset{a \mid U}{\bullet} \operatorname{ind}(a)=1^{[|U|-1]} \cdot(|U|+1) \text { or } \underset{a \mid U}{\bullet} \operatorname{ind}(a)=2^{[|U|]} .
$$

Suppose $n=2$. By Claim A, we see that $\operatorname{ind}(a)>1$ for each term $a \mid T$. Then $\underset{a \mid U}{\bullet \operatorname{ind}(a)=}$ $2^{[|U|]}$. Combined with (6), we have that $\operatorname{ind}(z) \geq 3$ and $\operatorname{ind}(z) \equiv 1(\bmod 2)$, where $z$ denotes the unique term of $W$. Combined with (7), then Condition (iii) holds.

Hence, it remains to consider the case of

$$
n \geq 3 \text {. }
$$

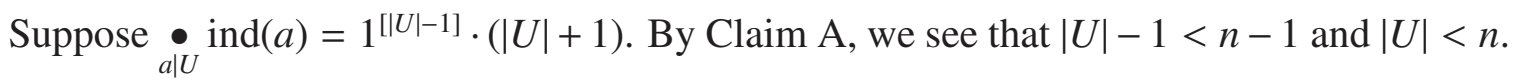
Since $2|U|=|U|-1+|U|+1=\sum_{a \mid U} \operatorname{ind}(a)$ is a positive multiple of $n$, it follows that $|U|=\frac{n}{2}$. Then all terms of $\Psi(T)$ are nonzero because neither of the two terms 1 and $|U|+1=\frac{n}{2}+1$ of the sequence $\underset{a \mid U}{\bullet} \operatorname{ind}(a)$ is congruent to 0 modulo $n$. By (6) and by applying Lemma 3.2 with $\Psi(T)$, we conclude that all terms of $\Psi(T)$ are equal, which is a contradiction with $1 \neq \frac{n}{2}+1=|U|+1$ $(\bmod n)$. Hence,

$$
\underset{a \mid U}{\bullet} \operatorname{ind}(a)=2^{[|U|]}
$$

It follows that all terms of $\Psi(T)$ are nonzero. By (6) and by applying Lemma 3.2 with $\Psi(T)$, we conclude that all terms of the sequence $\Psi(T)$ are equal to a generator of the group $\mathbb{Z} / n \mathbb{Z}$, i.e.,

$$
\operatorname{ind}(a) \equiv 2 \quad(\bmod n) \text { for each } a \mid W
$$

and

$$
n \equiv 1 \quad(\bmod 2) .
$$

Take an arbitrary term $\alpha$ of $W$ and an arbitrary term $\beta$ of $U$, and set $U^{\prime}=\left(U \cdot \beta^{[-1]}\right) \cdot \alpha$. It follows from (9) and (10) that $\Psi\left(U^{\prime}\right)$ is also a zero-sum sequence with $\left|U^{\prime}\right|=|U|$. By replacing $U$ with $U^{\prime}$ and by (9), we conclude that $\alpha=\beta=2$. By the arbitrariness of choosing $\alpha$, we have that

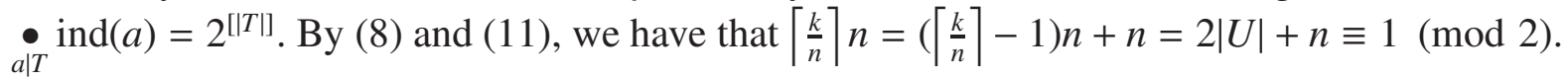
Combined with (7), then Condition (ii) holds. This completes the proof of the theorem.

Lemma 3.8. Let $\mathcal{S}=\mathrm{C}_{k ; n}$ with $1 \leq k \leq n$, and let $T \in \mathcal{F}(\mathcal{S})$ be a nonempty sequence. Then $T$ is an idempotent-sum free [resp. minimal idempotent-sum] sequence if and only if $\Psi(T) \in \mathcal{F}(\mathbb{Z} / n \mathbb{Z})$ is a zero-sum free [resp. minimal zero-sum] sequence.

Proof. Since $k \leq n$, we see that $\sum_{a \mid W} \operatorname{ind}(a) \equiv 0(\bmod n)$ implies $\sum_{a \mid W} \operatorname{ind}(a) \geq n=\left\lceil\frac{k}{n}\right\rceil n$ for any nonempty sequence $W \in \mathcal{F}(\mathcal{S})$. Then the lemma follows from Lemma 3.4 and the definition of the map $\Psi$ immediately. 
Lemma 3.9. ([14], Theorem 5.1.8) Let $G$ be a cyclic group of order $n \geq 3$. If $T \in \mathcal{F}(G)$ is zero-sum free of length at least $\left\lfloor\frac{n}{2}\right\rfloor+1$, then $T$ is $g$-smooth for some $g \in G$ with $\operatorname{ord}(g)=n$.

Now we are in a position to give the main theorem.

Theorem 3.10. For integers $k, n \geq 1$, let $T \in \mathcal{F}\left(\mathrm{C}_{k ; n}\right)$ be a sequence of length

$$
|T| \geq \begin{cases}\left\lfloor\frac{\left(\left\lceil\frac{k}{n}\right\rceil+1\right) n}{2}\right\rfloor, & \text { if } k>n ; \\ \left\lfloor\frac{n}{2}\right\rfloor+1, & \text { otherwise. }\end{cases}
$$

Then $T$ is idempotent-sum free if and only if one of the following two conditions holds:

(i) $\underset{a \mid T}{\bullet} \operatorname{ind}(a) \in \mathcal{F}(\mathbb{Z})$ is 1 -smooth with $\sum_{a \mid T} \operatorname{ind}(a) \leq\left\lceil\frac{k}{n}\right\rceil n-1$ in the case of $k>n$;

(ii) $\underset{a \mid T}{\bullet}(\operatorname{ind}(a)+n \mathbb{Z}) \in \mathcal{F}(\mathbb{Z} / n \mathbb{Z})$ is $g$-smooth for some $g \in \mathbb{Z} / n \mathbb{Z}$ with $\operatorname{ord}(g)=n$ in the case of $k \leq n$.

Proof. The sufficiency of the theorem follows from Definition 3.5 and Lemma 3.4.

For $k>n$, the necessity follows from Lemma 3.7 because the sequences meeting any one of Conditions (ii)-(v) have length exactly $\frac{\left(\left\lceil\frac{k}{n}\right\rceil+1\right) n}{2}-1<\left\lfloor\frac{\left(\left\lceil\frac{k}{n}\right\rceil+1\right) n}{2}\right\rfloor$.

Suppose $k \leq n$. By Lemma 3.8, then $\Psi(T)={ }_{a \mid T}^{\bullet}(\operatorname{ind}(a)+n \mathbb{Z}) \in \mathcal{F}(\mathbb{Z} / n \mathbb{Z})$ is a zero-sum free sequence with length $|\Psi(T)|=|T| \geq\left\lfloor\frac{n}{2}\right\rfloor+1$. Note that $n \geq 3$, since otherwise $n \in\{1,2\}$ then $\left\lfloor\frac{n}{2}\right\rfloor+1=n=\mathrm{D}(\mathbb{Z} / n \mathbb{Z})$ which is a contradiction with $\Psi(T)$ being zero-sum free. Then the necessity follows from Lemma 3.9 immediately.

Remark 3.11. We remark that the values $t$ in (12) of Theorem 3.10 are best possible in general to ensure an idempotent-sum free sequence $T$ over $\mathrm{C}_{k ; n}$ of length $|T| \geq t$ has the desired smooth sequence structure. The reason is as follows.

For $k>n$, we see that the sequence $T$ meeting any one of Conditions (ii)-(v) in Lemma 3.7 has length exactly $\left\lfloor\frac{\left(\left\lceil\frac{k}{n}\right\rceil+1\right) n}{2}\right\rfloor-1$ and satisfies $\underset{a \mid T}{\bullet} \operatorname{ind}(a)$ is not 1 -smooth. For $k \leq n$, one can check that the following idempotent-sum free sequence $V$ of length exactly $\left\lfloor\frac{n}{2}\right\rfloor$ and the sequence $\underset{a \mid V}{\bullet}(\operatorname{ind}(a)+n \mathbb{Z}) \in \mathcal{F}(\mathbb{Z} / n \mathbb{Z})$ is not smooth:

$$
\underset{a \mid V}{\bullet} \operatorname{ind}(a)=\left\{\begin{array}{lll}
1^{\left[\frac{n-5}{2}\right]} \cdot\left(\frac{n+3}{2}\right)^{[2]}, & \text { if } n \geq 8 \text { and } n \equiv 1 & (\bmod 2) \\
1^{\left[\frac{n-4}{2}\right]} \cdot\left(\frac{n+2}{2}\right)^{[2]}, & \text { if } n \geq 8 \text { and } n \equiv 0 & (\bmod 2)
\end{array}\right.
$$




\section{Concluding section}

Theorem 3.10 asserts that if an idempotent-sum free sequence $T$ over a cyclic semigroup $\mathrm{C}_{k ; n}$ has the length over 'approximately' a half of the size of the cyclic semigroup, then $T$ will have a smooth sequence structure. Although the quantities in (12) are best possible in general, it can be better for specific $k$ and $n$. So, one natural Ramsey-type question arises: For particular $k$ and $n$, what is the smallest positive integer $\ell$ such that every idempotent-sum free sequence $T$ over $\mathrm{C}_{k ; n}$ of length $|T| \geq \ell$ will yield a smooth sequence structure given as Theorem 3.10? This type of question has been investigated by S.T. Chapman, M. Freeze and W.W. Smith [3, 4], W. Gao [7], and P. Yuan [27] for minimal zero-sum sequences over finite cyclic groups, which is formulated as the invariant $\mathrm{I}(\cdot)$ below.

Definition A. ([14], Definition 5.1.1 and Lemma 5.1.2) Let $G$ be a cyclic group of order $n$.

(i) For any nonzero element $g \in G$ and for any sequence $T=\left(n_{1} g\right) \cdot \ldots \cdot\left(n_{\ell} g\right)$, where $\ell \in \mathbb{N} \cup\{0\}$ and $n_{1}, \ldots, n_{\ell} \in[1, \operatorname{ord}(g)]$, we define $\|T\|_{g}=\frac{n_{1}+\cdots+n_{\ell}}{\operatorname{ord}(g)}$;

(ii) For any $T \in \mathcal{F}(G)$, we call $\operatorname{ind}(T)=\min \left\{\|T\|_{g}: g \in G\right.$ with $\left.\operatorname{ord}(g)=n\right\} \in \mathbb{Q}_{\geq 0}$ the index of $T$;

(iii) Define $\mathrm{I}(G)$ to be the smallest integer $\ell \in \mathbb{N}$ such that every minimal zero-sum sequence $T \in \mathcal{F}(G)$ of length $|T| \geq \ell$ satisfies $\operatorname{ind}(T)=1$.

The invariant I $(\cdot)$ was completely determined by P. Yuan in a final critical step.

Theorem B. (see [27], or [[14], Corollary 5.1.9]) Let $G$ be a cyclic group of order $n \geq 1$. If $n \in\{1,2,3,4,5,7\}$ then $\mathrm{I}(G)=1$, and otherwise we have $\mathrm{I}(G)=\left\lfloor\frac{n}{2}\right\rfloor+2$.

Now we formulate two invariants on the Ramsey-type question associated with idempotentsum free and minimal idempotent-sum sequences over finite cyclic semigroups.

Definition 4.1. For $\max (k, n)>1$ we define $\operatorname{Smo}\left(\mathrm{C}_{k ; n}\right) \quad$ [resp. $\widehat{\operatorname{Smo}}\left(\mathrm{C}_{k ; n}\right)$ ] to be the least positive integer $\ell$ such that for any minimal idempotent-sum [resp. idempotent-sum free] sequence $T \in \mathcal{F}\left(\mathrm{C}_{k ; n}\right)$ of length at least $\ell$ satisfies:

(i) If $k>n$ then the sequence $\underset{a \mid T}{\bullet \operatorname{ind}}(a) \in \mathcal{F}(\mathbb{Z})$ is 1-smooth;

(ii) If $k \leq n$ then the sequence $\underset{a \mid T}{\bullet}(\operatorname{ind}(a)+n \mathbb{Z}) \in \mathcal{F}(\mathbb{Z} / n \mathbb{Z})$ is zero-sum $g$-smooth [resp. g-smooth] for some $g \in \mathbb{Z} / n \mathbb{Z}$ with $\operatorname{ord}(g)=n$.

We let $\operatorname{Smo}\left(C_{1 ; 1}\right)=1$ and $\widehat{\operatorname{Smo}}\left(C_{1 ; 1}\right)=0$. The following Lemma will illustrate us why the invariant $\operatorname{Smo}\left(\mathrm{C}_{k ; n}\right)$ coincides with $\mathrm{I}(\mathbb{Z} / n \mathbb{Z})$ for the case of $k \leq n$ with $n=6$ or $n \geq 8$.

Lemma 4.2. For $1 \leq k \leq n$, let $T \in \mathcal{F}\left(\mathrm{C}_{k ; n}\right)$ be a minimal idempotent-sum sequence. Then, 
(i) If $\underset{a \mid T}{\bullet}(\operatorname{ind}(a)+n \mathbb{Z}) \in \mathcal{F}(\mathbb{Z} / n \mathbb{Z})$ is zero-sum smooth then $\underset{a \mid T}{\operatorname{ind}(\bullet}(\operatorname{ind}(a)+n \mathbb{Z}))=1$;

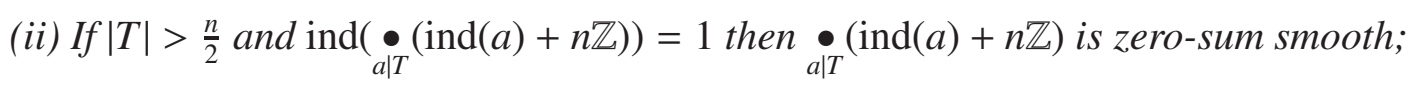

(iii) $\operatorname{Smo}\left(\mathrm{C}_{k ; n}\right) \geq \mathrm{I}(\mathbb{Z} / n \mathbb{Z})$, moreover, if $n=6$ or $n \geq 8$ then $\operatorname{Smo}\left(\mathrm{C}_{k ; n}\right)=\mathrm{I}(\mathbb{Z} / n \mathbb{Z})=\left\lfloor\frac{n}{2}\right\rfloor+2$.

Proof. (i). The conclusion follows from Definition 3.5 and Definition A.

(ii). Since ind $\underset{a \mid T}{\bullet}(\operatorname{ind}(a)+n \mathbb{Z}))=1$, it follows from Definition A that $\|\underset{a \mid T}{\bullet}(\operatorname{ind}(a)+n \mathbb{Z})\|_{g}=1$ for some $g \in \mathbb{Z} / n \mathbb{Z}$ with $\operatorname{ord}(g)=n$, i.e., $\underset{a \mid T}{\bullet}(\operatorname{ind}(a)+n \mathbb{Z})=\left(n_{1} g\right) \cdot \ldots \cdot\left(n_{t} g\right)$ where $t=|T|$,

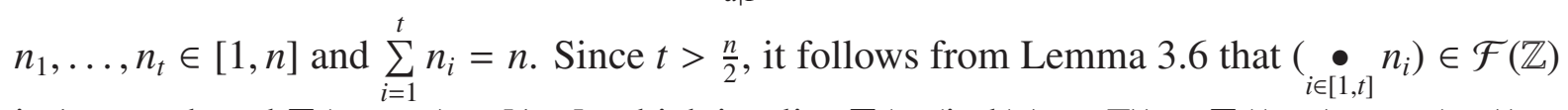
is 1 -smooth and $\sum\left(\underset{i \in[1, t]}{\bullet} n_{i}\right)=[1, n]$, which implies $\left.\sum \underset{a \mid T}{\bullet}(\operatorname{ind}(a)+n \mathbb{Z})\right)=\sum\left(\left(n_{1} g\right) \cdot \ldots \cdot\left(n_{t} g\right)\right)=$ $\{g, 2 g, \ldots, n g\}$. Hence, the sequence $\underset{a \mid T}{\bullet}(\operatorname{ind}(a)+n \mathbb{Z}) \in \mathcal{F}(\mathbb{Z} / n \mathbb{Z})$ is zero-sum $g$-smooth.

(iii). The conclusion $\operatorname{Smo}\left(\mathrm{C}_{k ; n}\right) \geq \mathrm{I}(\mathbb{Z} / n \mathbb{Z})$ follows from (i). Say $n=6$ or $n \geq 8$. By Theorem B, I $(\mathbb{Z} / n \mathbb{Z})=\left\lfloor\frac{n}{2}\right\rfloor+2>\frac{n}{2}$. Then $\operatorname{Smo}\left(C_{k ; n}\right)=\mathrm{I}(\mathbb{Z} / n \mathbb{Z})$ follows from (ii).

Together with the following observation, we shall have all ingredients to find the values of $\widehat{\operatorname{Smo}}\left(\mathrm{C}_{k ; n}\right)$ and $\operatorname{Smo}\left(\mathrm{C}_{k ; n}\right)$ for cyclic semigroups $\mathrm{C}_{k ; n}$.

Lemma 4.3. Let $H$ be a sequence of positive integers of length at least 2 , and let $h$ be one minimal term of $H$. If $H \cdot h^{[-1]}$ is 1-smooth, so is $H$.

Theorem 4.4. Let $k, n$ be positive integers. Then the following conclusions hold:

(i) For $k \leq n$, if $n=5$ then $\operatorname{Smo}\left(C_{k ; n}\right)=3$ and $\widehat{\operatorname{Smo}}\left(C_{k ; n}\right)=1$, and otherwise we have

$$
\operatorname{Smo}\left(C_{k ; n}\right)-1=\widehat{\operatorname{Smo}}\left(C_{k ; n}\right)= \begin{cases}\left\lfloor\frac{n}{2}\right\rfloor, & \text { if } n \leq 4 \text { or } n=7 \\ \left\lfloor\frac{n}{2}\right\rfloor+1, & \text { if } n=6 \text { or } n \geq 8\end{cases}
$$

(ii) For $k>n$, then $\operatorname{Smo}\left(\mathrm{C}_{k ; n}\right) \leq \widehat{\operatorname{Smo}}\left(\mathrm{C}_{k ; n}\right)+1$, moreover,

$$
\begin{cases}\frac{\left\lceil\frac{k}{n}\right\rceil n}{2}+1 \leq \widehat{\operatorname{Smo}}\left(C_{k ; n}\right) \leq\left\lceil\frac{\left(\left\lceil\frac{k}{n}\right\rceil+1\right) n}{2}\right\rceil-1, & \text { if } n \geq 3 \text { and }\left\lceil\frac{k}{n}\right\rceil n \equiv 0 \quad(\bmod 2) ; \\ \widehat{\operatorname{Smo}}\left(C_{k ; n}\right)=\left\lfloor\frac{\left(\left\lceil\frac{k}{n}\right\rceil+1\right) n}{2}\right\rfloor, & \text { otherwise, }\end{cases}
$$

and

$$
\begin{cases}\frac{\left\lceil\frac{k}{n}\right\rceil n}{2}+1 \leq \operatorname{Smo}\left(\mathrm{C}_{k ; n}\right) \leq\left\lceil\frac{\left(\left\lceil\frac{k}{n}\right\rceil+1\right) n}{2}\right\rceil, & \text { if } n \geq 3 \text { and }\left\lceil\frac{k}{n}\right\rceil n \equiv 0 \quad(\bmod 2) \\ \operatorname{Smo}\left(\mathrm{C}_{k ; n}\right)=\left\lfloor\frac{\left(\left\lceil\frac{k}{n}\right\rceil+1\right) n}{2}\right\rfloor, & \text { if } n=2 ; \\ \operatorname{Smo}\left(\mathrm{C}_{k ; n}\right)=\left\lfloor\frac{\left(\left\lceil\frac{k}{n}\right\rceil+1\right) n}{2}\right\rfloor+1, & \text { otherwise. }\end{cases}
$$


Proof. (i). By the definition, $\widehat{\operatorname{Smo}}\left(C_{1 ; 1}\right)=0$ and $\operatorname{Smo}\left(C_{1 ; 1}\right)=1$. Say $n \geq 2$. To calculate $\operatorname{Smo}\left(\mathrm{C}_{k ; n}\right)$, by Lemma 4.2 (iii), it remains to consider the case of $n \in\{2,3,4,5,7\}$. Take a sequence $W \in \mathcal{F}\left(\mathrm{C}_{k ; n}\right)$, where

$$
\underset{a \mid W}{\operatorname{ind}(a)}= \begin{cases}n, & \text { if } n \in\{2,3\} \\ 1 \cdot(n-1), & \text { if } n \in\{4,5\} \\ 1 \cdot 1 \cdot 5, & \text { if } n=7\end{cases}
$$

It is routine to check that $W$ is a minimal idempotent-sum sequence and the sequence $\underset{a \mid W}{\bullet}(\operatorname{ind}(a)+$ $n \mathbb{Z}) \in \mathcal{F}(\mathbb{Z} / n \mathbb{Z})$ is not zero-sum $g$-smooth for any $g \in \mathbb{Z} / n \mathbb{Z}$ with ord $(g)=n$, which implies

$$
\operatorname{Smo}\left(C_{k ; n}\right) \geq|W|+1=\left\lfloor\frac{n}{2}\right\rfloor+1 \text { for } n \in\{2,3,4,5,7\} .
$$

On the other hand, let $T \in \mathcal{F}\left(\mathrm{C}_{k ; n}\right)$ be a minimal idempotent-sum sequence such that

$$
|T| \geq\left\lfloor\frac{n}{2}\right\rfloor+1
$$

By Lemma 3.8 and Theorem B, see that ${ }_{a \mid T}^{\bullet}(\operatorname{ind}(a)+n \mathbb{Z}) \in \mathcal{F}(\mathbb{Z} / n \mathbb{Z})$ is a minimal zerosum sequence with ind $(\underset{a \mid T}{\bullet}(\operatorname{ind}(a)+n \mathbb{Z}))=1$. By (14) and Lemma 4.2 (ii), we derive that $\underset{a \mid T}{\bullet}(\operatorname{ind}(a)+n \mathbb{Z})$ is zero-sum smooth and so $\operatorname{Smo}\left(\mathrm{C}_{k ; n}\right) \leq\left\lfloor\frac{n}{2}\right\rfloor+1$, combined with (13), we have that $\operatorname{Smo}\left(\mathrm{C}_{k ; n}\right)=\left\lfloor\frac{n}{2}\right\rfloor+1$ where $n \in\{2,3,4,5,7\}$.

Next we figure out the value of $\widehat{\operatorname{Smo}}\left(\mathrm{C}_{k ; n}\right)$. If $n \leq 5$ or $n=7$, the conclusion follows by exhaustive but trivial calculations. If $n=6$ or $n \geq 8$, we see that the following idempotentsum free sequence $V \in \mathcal{F}\left(C_{k ; n}\right)$ has length exactly $\left\lfloor\frac{n}{2}\right\rfloor$ and $\underset{a \mid V}{\bullet}(\operatorname{ind}(a)+n \mathbb{Z}) \in \mathcal{F}(\mathbb{Z} / n \mathbb{Z})$ is not $g$-smooth for any $g \in \mathbb{Z} / n \mathbb{Z}$ with $\operatorname{ord}(g)=n$, which implies that $\widehat{\operatorname{Smo}}\left(C_{k ; n}\right) \geq|V|+1=\left\lfloor\frac{n}{2}\right\rfloor+1$, where

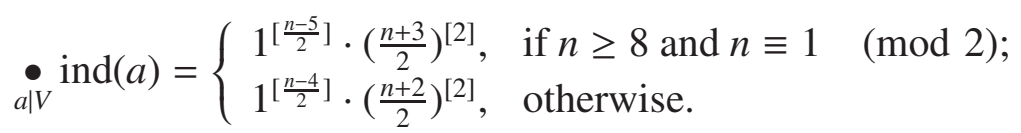

On the other hand, Theorem 3.10 tells us that $\widehat{\operatorname{Smo}}\left(\mathrm{C}_{k ; n}\right) \leq\left\lfloor\frac{n}{2}\right\rfloor+1$, and so $\widehat{\operatorname{Smo}}\left(\mathrm{C}_{k ; n}\right)=\left\lfloor\frac{n}{2}\right\rfloor+1$ when $n=6$ or $n \geq 8$, completing the calculations of $\widehat{\operatorname{Smo}}\left(C_{k ; n}\right)$.

(ii). We first calculate $\widehat{\operatorname{Smo}}\left(C_{k ; n}\right)$. Take a sequence $V \in \mathcal{F}\left(\mathrm{C}_{k ; n}\right)$ such that

$$
\underset{a \mid V}{\bullet} \operatorname{ind}(a)= \begin{cases}3 \cdot 2^{\left[\frac{\left[\frac{k}{n}\right\rceil n}{2}-1\right]}, & \text { if } n \geq 2 \text { and }\left\lceil\frac{k}{n}\right] n \equiv 0 \quad(\bmod 2) ; \\ 2^{\left.\left[\frac{\left.\left(\frac{k}{n}\right\rceil+1\right) n}{2}\right]-1\right]}, & \text { otherwise. }\end{cases}
$$

By Lemma 3.4, we can check that $V$ is an idempotent-sum free sequence. Since $\underset{a \mid V}{\bullet} \operatorname{ind}(a)$ is not 1-smooth, noting $\frac{\left\lceil\frac{k}{n}\right\rceil n}{2}+1=\left\lfloor\frac{\left(\left\lceil\frac{k}{n}\right\rceil+1\right) n}{2}\right\rfloor$ for $n=2$, we conclude that

$$
\widehat{\operatorname{Smo}}\left(C_{k ; n}\right) \geq|V|+1= \begin{cases}\frac{\left\lceil\frac{k}{n}\right\rceil n}{2}+1, & \text { if } n \geq 3 \text { and }\left\lceil\frac{k}{n}\right\rceil n \equiv 0 \quad(\bmod 2) \\ \left\lfloor\frac{\left(\left\lceil\frac{k}{n}\right\rceil+1\right) n}{2}\right\rfloor, & \text { otherwise }\end{cases}
$$


By Theorem 3.10, we derive that $\widehat{\operatorname{Smo}}\left(\mathrm{C}_{k ; n}\right) \leq\left\lfloor\frac{\left(\left\lceil\frac{k}{n}\right\rceil+1\right) n}{2}\right\rfloor$. Furthermore, if $n \geq 3$ and $\left\lceil\frac{k}{n}\right\rceil n \equiv 0$ (mod 2), we conclude from Lemma 3.7 that every idempotent-sum free sequence $U \in \mathcal{F}\left(\mathrm{C}_{k ; n}\right)$ of length at least $\frac{\left(\left\lceil\frac{k}{n}\right\rceil+1\right) n}{2}-1$ yields that $\underset{a \mid U}{\bullet} \operatorname{ind}(a)$ is 1 -smooth, i.e., $\widehat{\operatorname{Smo}}\left(\mathrm{C}_{k ; n}\right) \leq\left\lceil\frac{\left(\left\lceil\frac{k}{n}\right\rceil+1\right) n}{2}-1\right\rceil=$ $\left\lceil\frac{\left(\left\lceil\frac{k}{n}\right\rceil+1\right) n}{2}\right\rceil-1$. Combined with $(15)$, we complete the calculations of $\widehat{\operatorname{Smo}}\left(C_{k ; n}\right)$.

To establish $\operatorname{Smo}\left(C_{k ; n}\right) \leq \widehat{\operatorname{Smo}}\left(C_{k ; n}\right)+1$, let $T \in \mathcal{F}\left(C_{k ; n}\right)$ be a minimal idempotent-sum sequence of length at least $\widehat{\operatorname{Smo}}\left(\mathrm{C}_{k ; n}\right)+1$. It suffices to show that $\underset{a \mid T}{\bullet} \operatorname{ind}(a)$ is 1 -smooth. Take a term $b$ of $T$ with ind $(b)$ minimal. Since $T \cdot b^{[-1]}$ is an idempotent-sum free sequence of length $\left|T \cdot b^{[-1]}\right| \geq \widehat{\operatorname{Smo}}\left(\mathrm{C}_{k ; n}\right)$, it follows that $\underset{a \mid T \cdot b^{[-1]}}{\bullet} \operatorname{ind}(a)$ is 1-smooth. Then the conclusion follows from Lemma 4.3 immediately.

Now we calculate $\operatorname{Smo}\left(\mathrm{C}_{k ; n}\right)$. Take a sequence $W \in \mathcal{F}\left(\mathrm{C}_{k ; n}\right)$ with $\underset{a \mid W}{\bullet} \operatorname{ind}(a)=2^{[\ell]}$ and

$$
\ell= \begin{cases}\frac{\left\lceil\frac{k}{n}\right\rceil n}{2}, & \text { if }\left\lceil\frac{k}{n}\right\rceil n \equiv 0 \quad(\bmod 2) \\ \frac{\left(\left\lceil\frac{k}{n}\right\rceil+1\right) n}{2}, & \text { otherwise }\end{cases}
$$

By Lemma 3.4, we see that $W$ is a minimal idempotent-sum sequence. Since $\underset{a \mid W}{\bullet} \operatorname{ind}(a)$ is not 1-smooth, we have

$$
\operatorname{Smo}\left(C_{k ; n}\right) \geq|W|+1= \begin{cases}\frac{\left\lceil\frac{k}{n}\right\rceil n}{2}+1, & \text { if }\left\lceil\frac{k}{n}\right\rceil n \equiv 0 \quad(\bmod 2) \\ \frac{\left(\left\lceil\frac{k}{n}\right\rceil+1\right) n}{2}+1, & \text { otherwise }\end{cases}
$$

Noting that if $n=1$ and $k=\left\lceil\frac{k}{n}\right\rceil n \equiv 0(\bmod 2)$ then $\frac{\left\lceil\frac{k}{n}\right\rceil n}{2}+1=\left\lfloor\frac{\left(\left\lceil\frac{k}{n}\right\rceil+1\right) n}{2}\right\rfloor+1$ and that if $n=2$ then $\frac{\left\lceil\frac{k}{n}\right\rceil n}{2}+1=\left\lfloor\frac{\left(\left\lceil\frac{k}{n}\right\rceil+1\right) n}{2}\right\rfloor$, combined with the obtained inequality $\operatorname{Smo}\left(\mathrm{C}_{k ; n}\right) \leq \widehat{\operatorname{Smo}}\left(\mathrm{C}_{k ; n}\right)+1$ and the result for $\widehat{\operatorname{Smo}}\left(\mathrm{C}_{k ; n}\right)$, we conclude that

$$
\begin{cases}\frac{\left\lceil\frac{k}{n}\right\rceil n}{2}+1 \leq \operatorname{Smo}\left(C_{k ; n}\right) \leq\left\lceil\frac{\left(\left\lceil\frac{k}{n}\right\rceil+1\right) n}{2}\right\rceil, & \text { if } n \geq 3 \text { and }\left\lceil\frac{k}{n}\right\rceil n \equiv 0 \quad(\bmod 2) \\ \left\lfloor\frac{\left(\left\lceil\frac{k}{n}\right\rceil+1\right) n}{2}\right\rfloor \leq \operatorname{Smo}\left(C_{k ; n}\right) \leq\left\lfloor\frac{\left(\left\lceil\frac{k}{n}\right\rceil+1\right) n}{2}\right\rfloor+1, & \text { if } n=2 \\ \operatorname{Smo}\left(C_{k ; n}\right)=\left\lfloor\frac{\left(\left\lceil\frac{k}{n}\right\rceil+1\right) n}{2}\right\rfloor+1, & \text { otherwise }\end{cases}
$$

To complete the proof, it remains to show that $\operatorname{Smo}\left(\mathrm{C}_{k ; n}\right) \leq\left\lfloor\frac{\left(\left\lceil\frac{k}{n}\right\rceil+1\right) n}{2}\right\rfloor$ when $n=2$. Assume to the contrary that $\operatorname{Smo}\left(\mathrm{C}_{k ; n}\right)>\left\lfloor\frac{\left(\left\lceil\frac{k}{n}\right\rceil+1\right) n}{2}\right\rfloor$ for $n=2$. Take a minimal idempotent-sum sequence $L \in \mathcal{F}\left(\mathrm{C}_{k ; n}\right)$ of length $|L|=\operatorname{Smo}\left(\mathrm{C}_{k ; n}\right)-1 \geq\left\lfloor\frac{\left(\left\lceil\frac{k}{n}\right\rceil+1\right) n}{2}\right\rfloor$ such that $\underset{a \mid L}{\bullet} \operatorname{ind}(a)$ is not 1 -smooth. Take a term $b$ of $L$ with ind $(b)$ minimal. By Lemma 4.3, the sequence $\underset{a \mid L \cdot b^{[-1]}}{\bullet} \operatorname{ind}(a)$ is not 1smooth. Since $L \cdot b^{[-1]}$ is an idempotent-sum free sequence of length $\left|L \cdot b^{[-1]}\right| \geq\left\lfloor\frac{\left(\left[\frac{k}{n}\right\rceil+1\right) n}{2}\right\rfloor-1=$ 
$\frac{\left(\left\lceil\frac{k}{n}\right\rceil+1\right) n}{2}-1$, it follows from Lemma 3.7 that $\underset{a \mid L \cdot b b^{[-1]}}{\bullet} \operatorname{ind}(a)=z \cdot 2^{\left[\left\lceil\frac{k}{2}\right\rceil-1\right]}$ with $z \geq 3$ and $z \equiv 1$ $(\bmod 2)$. Since $\left\lceil\frac{k}{2}\right\rceil>1$, we can take a term $c$ of $L$ with $\operatorname{ind}(c)=2$. Combined with Lemma 3.4. we verify that $\sum_{a \mid L \cdot c^{-1]}} \operatorname{ind}(a)=2\left(\left\lceil\frac{k}{2}\right\rceil-2\right)+z+\operatorname{ind}(b) \geq 2\left(\left\lceil\frac{k}{2}\right\rceil-2\right)+3+1 \geq k$ and $\sum_{a \mid L \cdot c^{[-1]}} \operatorname{ind}(a)=\sum_{a \mid L} \operatorname{ind}(a)-2 \equiv \sum_{a \mid L} \operatorname{ind}(a) \equiv 0(\bmod 2)$, and so $L \cdot c^{[-1]}$ is a nonempty proper idempotent-sum subsequence of $L$, which contradicts with $L$ being a minimal idempotent-sum sequence, completing the proof.

We close this paper with the following problem.

Problem 4.5. Determine $\widehat{\operatorname{Smo}}\left(C_{k ; n}\right)$ and $\operatorname{Smo}\left(C_{k ; n}\right)$ when $k>n \geq 3$ and $\left\lceil\frac{k}{n}\right\rceil n \equiv 0(\bmod 2)$.

\section{Acknowledgements}

The research is supported by NSFC (grant no. 11971347, 11501561).

\section{References}

[1] J.D. Bovey, P. Erdős and I. Niven, Conditions for zero sum modulo n, Canad. Math. Bull., 18 (1975) 27-29.

[2] D.A. Burgess, A problem on semi-groups, Studia Sci. Math. Hungar., 4 (1969) 9-11.

[3] S.T. Chapman, M. Freeze and W.W. Smith, Minimal zero sequences and the strong Davenport constant, Discrete Math., 203 (1999) 271-277.

[4] S.T. Chapman and W.W. Smith, A characterization of minimal zero-sequences of index one in finite cyclic groups, Integers, 5 (2005) Paper A27, 5p.

[5] K. Cziszter, M. Domokos and A. Geroldinger, (2016) The Interplay of Invariant Theory with Multiplicative Ideal Theory and with Arithmetic Combinatorics. In: Chapman S., Fontana M., Geroldinger A., Olberding B. (eds) Multiplicative Ideal Theory and Factorization Theory, Springer Proceedings in Mathematics \& Statistics, vol 170. Springer, Cham.

[6] C. Deng, Davenport constant for commutative rings, J. Number Theory, 172 (2017) 321342.

[7] W. Gao, Zero sums in finite cyclic groups, Integers, 0 (2000) Paper A12, 7p.

[8] W. Gao and A. Geroldinger, On the structure of zerofree sequences, Combinatorica, 18 (1998) 519-527. 
[9] W. Gao and A. Geroldinger, On long minimal zero sequences in finite abelian groups, Period. Math. Hungar., 38 (1999) 179-211.

[10] W. Gao and A. Geroldinger, On zero-sum sequences in $\mathbb{Z} / n \mathbb{Z} \oplus \mathbb{Z} / n \mathbb{Z}$, Integers, 3 (2003) Paper A8, 45p.

[11] W. Gao, A. Geroldinger and D.J. Grynkiewicz, Inverse zero-sum problems III, Acta Arith., 141 (2010) 103-152.

[12] W. Gao, A. Geroldinger and W.A. Schmid, Inverse zero-sum problems, Acta Arith., 128 (2007) 245-279.

[13] W. Gao, Y. Li, P. Yuan and J. Zhuang, On the structure of long zero-sum free sequences and n-zero-sum free sequences over finite cyclic groups, Arch. Math., 105 (2015) 361370.

[14] A. Geroldinger, Additive Group Theory and Non-unique Factorizations, 1-86 in: A. Geroldinger and I. Ruzsa (Eds.), Combinatorial Number Theory and Additive Group Theory (Advanced Courses in Mathematics-CRM Barcelona), Birkhäuser, Basel, 2009.

[15] A. Geroldinger and F. Halter-Koch, Non-Unique Factorizations. Algebraic, Combinatorial and Analytic Theory, Pure Appl. Math., vol. 278, Chapman \& Hall/CRC, 2006.

[16] A. Geroldinger and Y.O. Hamidoune, Zero-sumfree sequences in cyclic groups and some arithmetical application, Journal de Théorie des Nombres de Bordeaux, 14 (2002) 221239.

[17] D.W.H. Gillam, T.E. Hall and N.H. Williams, On finite semigroups and idempotents, Bull. London Math. Soc., 4 (1972) 143-144.

[18] P.A. Grillet, Commutative Semigroups, Kluwer Academic Publishers, 2001.

[19] D.J. Grynkiewicz, Structural Additive Theory, Developments in Mathematics, vol. 30, Springer, Cham, 2013.

[20] C. Reiher, A proof of the theorem according to which every prime number posseses Property B, Ph.D. Dissertation, University of Rostock (2010).

[21] S. Savchev and F. Chen, Long zero-free sequences in finite cyclic groups, Discrete Math., 307 (2007) 2671-2679.

[22] W.A. Schmid, Inverse zero-sum problems II, Acta Arith., 143 (2010) 333-343.

[23] G. Wang, Davenport constant for semigroups II, J. Number Theory, 153 (2015) 124-134.

[24] G. Wang, Additively irreducible sequences in commutative semigroups, J. Combin. Theory Ser. A, 152 (2017) 380-397. 
[25] G. Wang, Structure of the largest idempotent-product free sequences in semigroups, J. Number Theory, 195 (2019) 84-95.

[26] H. Wang, L. Zhang, Q. Wang and Y. Qu, Davenport constant of the multiplicative semigroup of the quotient ring $\frac{\mathbb{F}_{p}[x]}{\langle f(x)\rangle}$, International Journal of Number Theory, 12 (2016) 663669.

[27] P. Yuan, On the index of minimal zero-sum sequences over finite cyclic groups, J. Combin. Theory Ser. A, 114 (2007) 1545-1551.

[28] L. Zhang, H. Wang and Y. Qu, A problem of Wang on Davenport constant for the multiplicative semigroup of the quotient ring of $\mathbb{F}_{2}[x]$, Colloq. Math., 148 (2017) 123-130. 